This item was submitted to Loughborough's Research Repository by the author.

Items in Figshare are protected by copyright, with all rights reserved, unless otherwise indicated.

\title{
Mechanisms of copper protrusion in through-silicon-via structures at the nanoscale
}

PLEASE CITE THE PUBLISHED VERSION

https://doi.org/10.7567/1347-4065/aae898

\section{PUBLISHER}

IOP Publishing $@$ The Japan Society of Applied Physics

\section{VERSION}

AM (Accepted Manuscript)

\section{PUBLISHER STATEMENT}

This is an author-created, un-copyedited version of an article published in Journal of Applied Physics. IOP Publishing Ltd is not responsible for any errors or omissions in this version of the manuscript or any version derived from it. The Version of Record is available online at https://doi.org/10.7567/1347-4065/aae898.

\section{LICENCE}

\section{All Rights Reserved}

\section{REPOSITORY RECORD}

Liu, Jinxin, Zhiheng Huang, Yong Zhang, and Paul Conway. 2019. "Mechanisms of Copper Protrusion in Through-silicon-via Structures at the Nanoscale". figshare. https://hdl.handle.net/2134/35406. 


\section{Mechanisms of Copper Protrusion in Through-silicon-via Structures at the Nanoscale}

Jinxin Liu ${ }^{1}$, Zhiheng Huang ${ }^{1 *}$, Yong Zhang ${ }^{2 *}$ and Paul P. Conway ${ }^{3}$

${ }^{1}$ School of Materials Science and Engineering, Sun Yat-sen University, Guangzhou 510275, China

${ }^{2}$ School of Physics, Sun Yat-sen University, Guangzhou 510275, China

${ }^{3}$ School of Mechanical, Electrical and Manufacturing Engineering, Loughborough University, Loughborough, Leicestershire, LE11 3TU, UK

*E-mail: hzh29@mail.sysu.edu.cn, zhyong9@mail.sysu.edu.cn

Thermal stress-induced copper protrusion is frequently observed in through-silicon-vias (TSVs) based three-dimensional (3D) system integration. In this study, the detailed process of $\mathrm{Cu}$ protrusion is reproduced on the atomic scale using a two-mode phase-field-crystal (PFC) model, and the mechanisms of protrusion are identified. To simulate thermal loading, a "penalty term" is added to the governing equation of the PFC model. The application of loading on the TSVs induces copper grain deformation and grain boundary migration at the nanoscale. Furthermore, the simulation results suggest that the $\mathrm{Cu}$ protrusion is resulted from diffusional creep, involving both Nabarro-Herring creep and Coble creep. The obtained power index of diffusional creep $p$ is around 2.16, suggesting that lattice diffusion contributes more to protrusion than grain boundary diffusion does. The protrusion height in micron-scale TSVs predicted by extrapolating the relationship between the protrusion height and diameter of nanoscale TSVs agrees with the experimental data. 


\section{Introduction}

There-dimensional (3D) system integration provides a solution to bridge the gap between the slowing of Moore's law on scaling and the increasing package density requirements. An important idea in 3D system integration is to use through-silicon-via (TSV) technology, which enables multi-chip stacking in a vertical direction and is being widely used in 3D electronics devices. ${ }^{1-3)}$ As for examples, the mass production of TSV-based DDR4 memory was started ${ }^{4)}$ and the first GPU that utilized TSVs to mount high bandwidth memory (HBM) on silicon interposer was also reported. ${ }^{5)}$ The TSV structure is fabricated by filling electroplated copper into an etched silicon via. However, the mismatch of the coefficient of thermal expansion of the filler material, i.e., copper, and the surrounding silicon induces thermal stress in the TSV structure during thermal processing. ${ }^{6)}$ As a result, the Cu protrusion out of the TSV surface is observed. This causes the damage of back-end-of-line (BEOL) layers in 3D packaging and poses an urgent problem to be solved in the practical application of TSVs.

Extensive work involving experiments and simulations has been conducted to identify the mechanisms of $\mathrm{Cu}$ protrusion, but the root cause remains unclear. Messemaeker et al. reported that the $\mathrm{Cu}$ protrusions exhibited a statistical distribution and smaller protrusions were found in TSVs annealed at higher temperatures and for longer times. ${ }^{7)}$ Zhang et al. also found that Cu protrusions varied significantly from TSV to TSV and had random peaks on a $300 \mathrm{~mm}$ diameter wafer. ${ }^{8)}$ This statistical and random nature of the protrusion distribution reflects the effects from the microstructure of copper. ${ }^{9)}$ In addition, there exist a few experimental techniques to investigate the stress state in the TSV structures, e.g., a synchrotron X-ray microdiffraction technique used by Liu et al. ${ }^{10)}$ and a photo-elastic microscopy technique used by Herms et al. ${ }^{11)}$ As for simulations, a finite element method (FEM)-based thermal-mechanical modeling was used to predict the protrusion height by Che et al. ${ }^{12)}$ However, no further information on the effects of microstructure on $\mathrm{Cu}$ protrusion was provided in the aforementioned studies. Meanwhile, as 3D electronic devices and interconnects approach the nanoscale, ${ }^{13)}$ the material microstructure becomes a significant reliability concern. ${ }^{9,14-15)}$ On investigations into the microstructure of TSVs, Jiang et al. used an electron back-scattered diffraction (EBSD) technique and showed that larger grains presented in TSVs under thermal cycling with a higher peak temperature. ${ }^{14)}$ Recently, a large ensemble of $\mathrm{Cu}$ protrusions were examined and a statistical model correlating the protrusion height and the number of grains near the TSV top end was established. ${ }^{15)}$ Nevertheless, none of the above mentioned research revealed the evolution of microstructure in TSV during the 
protrusion process, which is yet critical to elucidate the root cause for the $\mathrm{Cu}$ protrusion.

To address this critical issue, we attempted to reproduce the process of $\mathrm{Cu}$ protrusion on the atomic scale using a phase-field-crystal (PFC) model. ${ }^{16)}$ To simulate the process of protrusion, the PFC model is more efficient than the molecular dynamics (MD) method since MD is limited to phonon time scale while PFC can access diffusive time scale. ${ }^{17)}$ In addition, the PFC model naturally incorporates the elastic and plastic behavior of crystals, e.g., the movement of defects such as dislocations and grain boundaries (GBs). ${ }^{17-19)}$ This work aims to present further analysis and insight into the mechanisms of $\mathrm{Cu}$ protrusion in TSV structures at the nanoscale. The atomic scale microstructure evolution of polycrystalline copper and the resulting protrusion in TSV structures under thermal loading is revealed from the PFC model. Then, a diffusional creep model is used to explain the mechanisms of $\mathrm{Cu}$ protrusion in detail.

\section{Methods}

In the PFC model, an order parameter $\rho$, which has the form of a plane wave with the same periodicity as the lattice, is introduced to represent the local time-averaged atomic density of a material system. Furthermore, using a two-point correlation function, a two-mode PFC model has been developed to simulate a square lattice in two dimensions. ${ }^{20)}$ In the $2 \mathrm{D}$ case, the two-mode order parameter is written as: ${ }^{20)}$

$$
\rho=\bar{\rho}+A[\cos (q x)+\cos (q y)]+B \cos (q x) \cos (q y)
$$

where $A$ and $B$ are related to the amplitudes of two sets of density waves. $q$ is a parameter related to the lattice constant, hereinafter referred to as $a$, through the equation $q=2 \pi / a$. In the $2 \mathrm{D}$ simulation, a square lattice corresponds to the $\{100\}$ crystallographic plane of copper. Then, the free energy of the system is written as: ${ }^{20)}$

$$
F[\rho]=\int \mathrm{d} \vec{r}\left\{\frac{\rho}{2}\left[r+\left(\nabla^{2}+1\right)^{2}\left(\nabla^{2}+Q^{2}\right)^{2}\right] \rho+\frac{\rho^{4}}{4}\right\}
$$

where $r=c\left(T-T_{\mathrm{m}}\right) / T_{\mathrm{m}}$ is the scaling temperature and $Q^{2}=2$ is a constant. Based on the first variation in the free energy of the system with respect to the order parameter, the evolution of the microstructure is obtained by solving the governing equation: ${ }^{20}$

$$
\frac{\partial \rho}{\partial t}=\nabla^{2} \frac{\delta F}{\delta \rho}
$$

Due to the geometry symmetry of the TSV, a 2D PFC model is adopted in this study for computational efficiency. The model configuration of our TSV structure is shown in Fig. 1a. A trapezoid TSV is chosen because of the tapered nature of a drilled via, ${ }^{21)}$ and the geometry effect was studied in our previous work. ${ }^{22)}$ The edges of the TSV are denoted by solid gray 
lines. The atomic-scale microstructure is developed by placing crystal nuclei inside the TSV and then inducing solidification. The microstructure is further equilibrated until it remains unchanged. Notably, the $\mathrm{Cu}$ filling is performed via an electroplating process rather than a solidification process. The grain structures generated and equilibrated through the PFC model are carefully controlled by referring to representative grain structures in the TSVs characterized via EBSD to investigate the mechanisms of $\mathrm{Cu}$ protrusion. To apply a mechanical loading, external layers of crystals are added to surround the TSV. The top layer is set as a grain with a $0^{\circ}$ orientation, providing a solid cover for the TSV. The grain orientations of other external layers are adjusted to accommodate the corresponding grains at both edges of the TSV. Then, a "penalty term" is added to the free energy with the form: ${ }^{19)}$

$$
F[\rho]^{\prime}=F[\rho]+\int \mathrm{d} \vec{r} M(\vec{r})[\rho(\vec{r}, t)-\rho(\vec{r}(\vec{v}, t), t)]
$$

where $M(\vec{r})$ is nonzero only in the external layers, except for the top cover layer. With the "penalty term" applied, the atoms inside the external layers are motivated to move with a predetermined velocity $\vec{v}\left(|\vec{v}|=1.0 \times 10^{-4}\right)$ in a direction defined by the arrows, forming an angle $\theta=150^{\circ}$ with the horizontal line, as demonstrated in Fig. 1a. The "penalty term" mimics the application of a compressive strain to the TSV. Further description on the applied loading to the TSV is illustrated in Fig. 1. Note that the strain state considered here refers to results from experimental work and FEM simulations, where a compressive strain in the TSV has been observed. ${ }^{10,23-24)}$ The applied strain can be decomposed into two components, a compressive strain and a shear strain, along the edge of the TSV. The loading is assumed to be uniformly distributed along the edge for simplicity. Other parameters used in the PFC model are set as $(r, \bar{\rho}, A, B, q)=(-1,0.59,-0.31,-0.14,1.0)$. The numerical parameters are determined by minimizing the free energy functional in Equation (2). ${ }^{19)}$ More details about the parameters are referred to elsewhere. ${ }^{19-20)}$ Note that the length and time scales in the original PFC model and this study are dimensionless. In addition, the atoms in or near the GBs and dislocations in the TSV are defined as defect atoms, as highlighted by the yellow dots in Fig. 1a.

\section{Results and discussion}

From the initial solidified and equilibrated microstructure shown in Fig. 1a, the black dashed curve in Fig. 1c plots the protrusion profile of the TSV at time $t=30000$ during the loading stage. Here, the applied strain is around $10^{-3}$, which is of the same order of magnitude as the strain in TSV measured from a synchrotron X-ray microdiffraction. ${ }^{10)}$ This protrusion profile is similar to experimental results in the literature, ${ }^{25-26)}$ presenting a random surface 
profile of the top end of the TSV. The randomness in the protrusion profile is not well reproduced using FEM models. In contrast, the profiles predicted with FEM models are generally one-mode sinusoids and symmetric to the vertical center axis of the TSV because the copper is treated as a bulk, isotropic material in FEM models. ${ }^{27-29)}$

Fig. 2a-2b demonstrate the movement of a dislocation under applied strain. The dislocation climbs a distance of approximately $2 a$ from $t=8000$ to $t=9000$ during the protrusion process. Then, the dislocation moves to the top end of the TSV, resulting in a bulge along the GB, as shown in Fig. 2c, in which the defect atoms are highlighted. In addition, grain deformation and GB migration are observed. For example, comparing Fig. 1c to Fig. 1a, grains 1 and 2 elongate in the vertical direction. To achieve such a configuration, the GBs of the two grains must migrate. As shown in Figs. 2c-2d, the GB between grain 1 and the top cover layer migrates upwards from $t=10000$ to $t=20000$. The same process is also observed for the GB between grain 2 and the top cover layer. Although the cover layer in a real case is somewhat different, i.e., usually a copper line with a TiN encapsulation barrier, ${ }^{30-32)}$ the abovementioned atomic-scale processes would remain similar.

Four TSVs exhibiting different grain structures, labeled TSV1 to TSV4, are shown in Fig. 3. In general, the four TSVs contain three grain layers, denoted the top, middle and bottom layer. The top layer contains two grains in all structures, whereas the middle and bottom layers contain different numbers of grains. For example, TSV2 contains three grains in the middle layer and one grain in the bottom layer, and its initial microstructure is shown in Fig. 1a. The protrusion profiles of the TSVs after strain is applied for $\Delta t=30000$ are sketched by the black dashed curves in Fig. 3, and the maximum protrusion height data are plotted in the same graph. TSV1 is found to protrude the least, and protrusion increases with the number of grains present in the middle layer, as evidenced from TSV1 to TSV3. The different protrusion heights observed for the different TSVs can be explained by the inhibition of GB migration between the grains in the top and middle layers. More grains in the middle layer increase the opportunity for three grains to join, forming a triple junction, as highlighted by the dashed circle in TSV3. The mobility of the triple junction is sufficiently low, ${ }^{33)}$ and once triple junctions are formed, the GBs between the grains in the top and middle layers cannot move as easily. Thus, deformation along the vertical direction of the two grains in the top layer is inhibited by the presence of triple junctions in the middle layer. With the accumulation of applied strain, more protrusion out of the top end of the TSV results. An experiment by Messemaeker et al. ${ }^{15)}$ shows that the protrusion height is related to the number of grains about $2.1 \mu \mathrm{m}$ below the TSV top surface. TSVs containing more grains below the 
top surface exhibit higher $\mathrm{Cu}$ protrusion. ${ }^{15)}$ In addition, TSV3 and TSV4 show almost the same protrusion, which suggests that the number of grains in the bottom layer of the TSV has little effect on protrusion. For the case discussed above, GB migration in the TSV controls grain deformation, especially for the grains in the top layer, which directly leads to the protrusion.

The trajectories of the atoms inside the TSV were analyzed to further investigate the microstructural evolution. In particular, the atom movements that led to the protrusion in Fig. 4a were recorded and are shown in Fig. 4b. A trajectory connects the initial location of an atom at $t=0$ to its corresponding location at $t=30000$. Clearly, the atoms collectively diffuse upwards. Therefore, most of the grains in the TSV also move upwards, and protrusion directly results.

The deformation of the grains in the TSVs can be described by the power law of diffusional creep. ${ }^{34-35)}$ A diffusional creep model has been adopted by Messemaeker et al. to predict the effect of TSV diameters on the Cu protrusion and the model has been validated by experimental measurement. ${ }^{7)}$ The deformation of material under diffusional creep always results from the diffusion of atoms or vacancies and is described by the following equation: ${ }^{35)}$

$$
\dot{\varepsilon} \propto C\left(\frac{1}{d}\right)^{p}
$$

where $\dot{\varepsilon}$ is the strain rate, $C$ is a constant related to the stress and $d$ is the average diameter of the grains. $p$ is a power index associated with the diffusional creep mechanism, e.g., $p=$ 2 for Nabarro-Herring creep and $p=3$ for Coble creep.

To investigate the relationship between the strain rate and grain size, the average grain size in the TSV was changed systematically from approximately $20 a$ to $40 a$. Then, the strain of the grains in the top layer and the strain rate were calculated. As shown in Fig. 5, the relationship between $\ln \dot{\varepsilon}$ and $\ln \left(d^{-1}\right)$ is approximately linear. Then, loadings along different directions, i.e., $\theta=180^{\circ}, 150^{\circ}$ and $120^{\circ}$, were applied to the TSV. The corresponding fitted slopes of the relationship between $\ln \dot{\varepsilon}$ and $\ln \left(d^{-1}\right)$, i.e., the power indices $p$, are 2.35, 1.78 and 2.37, respectively. The obtained power index suggests that the creep mechanism leading to protrusion may involve Nabarro-Herring creep and Coble creep. The contribution from lattice diffusion here is larger than GB diffusion. To further confirm the mechanism, the relationship between the scaling temperature $r$ and the real temperature must be determined. This is achieved by systematically varying $r$ and recording the corresponding glide velocity of dislocations in the PFC models. Then, the relationship between the velocity of dislocations and temperature $v=v_{0} e^{-\Delta G / k_{\mathrm{B}} T}$ is used. ${ }^{36)}$ Following this relationship, the system temperature is determined to be approximately $990 \mathrm{~K}$ and higher 
than the post-plating annealing temperature of the TSV. Despite this difference in temperature, the deformation mechanism remains the same. It has been reported that diffusional creep is the dominant mechanism in the temperature range between $600 \mathrm{~K}$ and $1100 \mathrm{~K}$ according to molecular dynamics simulations. ${ }^{37-38)}$ Coble creep dominates at lower temperatures and there exists a transition to Nabarro-Herring creep as temperature increases. ${ }^{39-41)}$ Moreover, the deformation mechanism of nanograined copper at $960 \mathrm{~K}$ has also been verified as diffusional creep with $p=2.21 \pm 0.44 .^{42)}$ The effect of temperature on the mechanism of $\mathrm{Cu}$ protrusion will be further investigated in a future study.

In addition, the deformation of the grains, i.e., diffusional creep, can be considered as the diffusion of the order parameter $\rho$. In the PFC model, the free energy functional can be rewritten as $F[\rho]=F_{i d}[\rho]+F_{e x}[\rho]$, where $F_{i d}[\rho]=\int d \vec{r}\left\{\frac{\rho^{2}}{2} r+\frac{\rho^{4}}{4}\right\}$ and $F_{e x}[\rho]=$ $\int d \vec{r}\left\{\frac{\rho}{2}\left(\nabla^{2}+1\right)^{2}\left(\nabla^{2}+Q^{2}\right)^{2} \rho\right\}$. The governing equation of the PFC model is therefore transformed to

$$
\frac{\partial \rho}{\partial t}=\nabla^{2} \frac{\delta F}{\delta \rho}=\nabla^{2} \frac{\delta\left(F_{i d}[\rho]+F_{e x}[\rho]\right)}{\delta \rho}=\nabla^{2}\left\{r \rho+\rho^{3}+\left(\nabla^{2}+1\right)^{2}\left(\nabla^{2}+Q^{2}\right)^{2} \rho\right\}
$$

It is noted that the first two terms on the right hand side of Equation (6) derives from $F_{i d}[\rho]$, while the rest of the terms there derives from $F_{e x}[\rho]$. From the numerical point of view, the term $F_{i d}[\rho]$ in Equation (2) may contribute to the diffusional creep. Because the diffusion term in a partial differential equation contains only a second derivative of the field.

Due to the computationally-intensive nature of PFC models, we focus on nanoscale TSV structures in this study. Although the scale of the investigated TSV structures is limited, the atomic-scale mechanisms resulting in protrusion remain the same for micron-scale structures. In fact, the extrapolated relationship between the average protrusion height and the TSV size, as shown in Fig. 6, predicts the protrusion to be $400 \mathrm{~nm}$ for a TSV with a diameter of approximately $5 \mu \mathrm{m}$. This is comparable to the experimental results that are obtained when TSVs are subjected to a high-temperature $\left(400^{\circ} \mathrm{C}\right)$ annealing..$^{8,12,14,26)}$

\section{Conclusions}

This study used a two-mode PFC model to reproduce the detailed process of $\mathrm{Cu}$ protrusion in TSV structures at the nanoscale. It has been found that GB migration contributes to and controls the protrusion. The collectively upward diffusion of atoms is also observed under the applied loading. Moreover, the mechanism of $\mathrm{Cu}$ protrusion can be explained by the power law of diffusional creep. The obtained power index suggests that lattice diffusion has more contribution to protrusion than GB diffusion does. In addition, the protrusion height in 
micron-scale TSVs predicted by extrapolating the relationship between the protrusion height and diameter of nanoscale TSVs agrees with the experimental data.

Throughout this study, understanding of the mechanism of $\mathrm{Cu}$ protrusion, at the atomic scale in particular, has been deepened. Methods to alleviate the $\mathrm{Cu}$ protrusion can be deduced from our simulation results, for examples, adding additives in the electrodeposited copper to inhibit the GB migrations or increasing the grain size in the TSVs. The authors believe that the effort to understand the $\mathrm{Cu}$ protrusion mechanisms at the atomic scale will shed a new light on the design of reliability of TSV-based 3D electronics devices.

\section{Acknowledgments}

The authors acknowledge financial support from the National Natural Science Foundation of China (NSFC) under grants 51004118 and 51832002, the Pearl River Science and Technology Nova Program of Guangzhou under grant 2012J2200074, and the Guangdong Natural Science Foundation under grant 2015A030312011. Financial support from YuxiTech (Guangzhou) Limited is also gratefully acknowledged. The authors thank Dr. Hua Lu of the University of Greenwich for helpful discussions and the anonymous reviewer for his comments to improve the quality of the manuscript. 


\section{References}

1) M.M. Shulaker, G. Hills, R.S. Park, R.T. Howe, K. Saraswat, H.S.P. Wong, and S. Mitra, Nature 547, 74 (2017).

2) W.W. Shen, and K.N. Chen, Nanoscale Res. Lett. 12[1], 56 (2017).

3) J.P. Gambino, S.A. Adderly, J.U. Knickerbocker, Microelectron. Eng. 135, 73 (2015).

4) R. Oh, B. Lee, S.-W. Shin, W. Bae, H. Choi, I. Song, Y.-S. Lee, J.-H. Choi, C.-W. Kim, S.J. Jang, J.-S. Choi, Symposium on VLSI Circuits Digest of Technical Papers, 2014 p. 1-2.

5) C.-C. Lee, C. Hung, C. Cheung, P.-F. Yang, C.-L. Kao, D.-L. Chen, M.-K. Shih, C.-L. C. Chien, Y.-H. Hsiao, L.-C. Chen, M. Su, M. Alfano, J. Siegel, J. Din and B. Black, Proc. IEEE Electronic Components and Technology Conference, 2016, p. 1439-1444.

6) D. Vogel, E. Auerswald, J. Auersperg, P. Bayat, R. Rodriguez, D. Zahn, S. Rzepka, and B. Michel, Microelectron. Reliab. 54[9-10], 1963 (2014).

7) J. De Messemaeker, O.V. Pedreira, B. Vandevelde, H. Philipsen, I. De Wolf, E. Beyne, and K. Croes, Proc. Electronic Components and Technology Conference, 2013, p. 586-591.

8) D. Zhang, K. Hummler, L. Smith, and J.J.Q. Lu, Proc. Electronic Components and Technology Conference, 2013, p. 1407-1413.

9) C. Okoro, L.E. Levine, R. Xu, and Y.S. Obeng, Proc. IEEE Electronic Components and Technology Conference, 2015, p. 54-58.

10) X. Liu, P.A. Thadesar, C.L. Taylor, H. Oh, M. Kunz, N. Tamura, M.S. Bakir, and S.K. Sitaraman, Appl. Phys. Lett. 105[11], 112109 (2014).

11) H. Martin, W. Matthias, D.M. Joke, and D.W. Ingrid, Phys. Status Solidi C 14[7], 1700028 (2017).

12) F.X. Che, W.N. Putra, A. Heryanto, A. Trigg, X. Zhang, and C.L. Gan, IEEE Trans. Compon., Packag., Manuf. Technol. 3[5], 732 (2013).

13) W.H. Xu, L. Wang, Z. Guo, X. Chen, J. Liu, and X.J. Huang, ACS Nano 9[1], 241 (2015).

14) T. Jiang, J. Im, R. Huang, and P.S. Ho, MRS Bull. 40[3], 248 (2015).

15) J.D. Messemaeker, P.J. Roussel, O.V. Pedreira, T.V. der Donck, S.V. Huylenbroeck, E. Beyne, I.D. Wolf, M. Stucchi, and K. Croes, IEEE Trans. Device Mater. Reliab. 17[3], 549 (2017).

16) J. Liu, Z. Huang, P.P. Conway, F. Altmann, M. Petzold, and F. Naumann, Proc. Int. Conference on Electronic Packaging Technology, 2017, p. 789-796.

17) K.R. Elder, M. Katakowski, M. Haataja, and M. Grant, Phys. Rev. Lett. 88, 245701 (2002).

18) J. Berry, M. Grant, and K.R. Elder, Phys. Rev. E 73, 031609 (2006).

19) P. Stefanovic, M. Haataja, and N. Provatas, Phys. Rev. E 80, 046107 (2009). 
20) K.A.Wu, A. Adland, and A. Karma, Phys. Rev. E 81, 061601 (2010).

21) S. Spiesshoefer, Z. Rahman, G. Vangara, S. Polamreddy, S. Burkett, and L. Schaper, J. Vac. Sci. Technol., A 23[4], 824 (2005).

22) Z. Huang, J. Liu, P.P. Conway, Z. Hu, and Y. Liu, Proc. Electronic System-Integration Technology Conference, 2016, p. 1-8.

23) E. Cheng, and Y.L. Shen, Microelectron. Reliab. 52[3], 534 (2012).

24) X. Liu, Q. Chen, P. Dixit, R. Chatterjee, R.R. Tummala, and S.K. Sitaraman, Proc. Electronic Components and Technology Conference, 2009, p. 624-629.

25) A. Heryanto, W. Putra, A. Trigg, S. Gao, W. Kwon, F. Che, X. Ang, J. Wei, R. I Made, C. Gan, and K. Pey, J. Electron. Mater. 41[9], 2533 (2012).

26) T. Jiang, C. Wu, L. Spinella, J. Im, N. Tamura, M. Kunz, H.Y. Son, B.G. Kim, R. Huang, and P.S. Ho, Appl. Phys. Lett. 103[21], 211906 (2013).

27) S.K. Ryu, T. Jiang, K.H. Lu, J. Im, H.Y. Son, K.Y. Byun, R. Huang, and P.S. Ho, Appl. Phys. Lett. 100[4], 041901 (2012).

28) H.S. Jung, Y.J. Jang, S.H. Choa, and J.P. Jung, Mater. Trans. 56[12], 2034 (2015).

29) D. Malta, C. Gregory, M. Lueck, J. Lannon, J. Lewis, D. Temple, P. DiFonzo, F. Naumann, and M. Petzold, Proc. Electronic Components and Technology Conference, 2013, p. 22352242.

30) T. Frank, S. Moreau, C. Chappaz, L. Arnaud, P. Leduc, A. Thuaire, and L. Anghel, Proc. IEEE Electronic Components and Technology Conference, 2012, p. 326-330.

31) Z. Chen, Z. Lv, X. Wang, and Yong, Proc. Int. Conference on Electronic Packaging Technology High Density Packaging, 2010, p. 1221-1225.

32) J. Pak, M. Pathak, S.K. Lim, and D.Z. Pan, Proc. IEEE Electronic Components and Technology Conference, 2011, p. 1420-1427.

33) A. Rollett, F. Humphreys, G. Rohrer, and M. Hatherly, Recrystallization and Related Annealing Phenomena (Elsevier, 2004), 2nd ed., p. 165-167

34) B. Wilshire, and A. Battenbough, Mater. Sci. Eng. A 443[12], 156 (2007).

35) J. Berry, J. Rottler, C.W. Sinclair, and N. Provatas, Phys. Rev. B 92, 134103 (2015).

36) G. Gottstein, Physical foundations of materials science (Springer Science \& Business Media, 2013).

37) Y.J. Wang, A. Ishii, and S. Ogata, Phys. Rev. B 84[22], 224102 (2011).

38) Y.J. Wang, G.J.J. Gao, and S. Ogata, Phys. Rev. B 88[11], 115413(2013).

39) M. Kawasaki, and T.G. Langdon, J. Mater. Res. 28[13], 1827 (2013).

40) A.H. Chokshi, Scr. Mater. 34[12], 1905 (1996). 
41) F.A. Mohamed, and Y. Li, Mater. Sci. Eng. A 298[1-2], 1 (2001).

42) Y.J. Wang, A. Ishii, and S. Ogata, Mater. Trans. 53[1], 156 (2012). 


\section{Figure Captions}

Fig. 1. (Color online) (a) The model configuration of a TSV structure. The edges of a trapezoid TSV at $t=0$ are outlined by solid gray lines. The parallel arrows indicate the direction of the strain applied to the TSV, which form an angle $\theta$ with the horizontal line. The defect atoms in the TSV are highlighted by the yellow dots. Note that the surface of TSV is not absolutely flat in (a) at the beginning of simulation, which is the result from a structure equilibration process between the TSV and the surrounding layers. To illustrate the applied loading, (b) and (c) show the snapshots of the TSV at $t=15000$ and $t=30000$ during the loading stage, respectively. The atoms in the external layers surrounding the left and right edges are highlighted by red dots. The red atoms are motivated to move by the "penalty term”, thus giving the effect of a compressive strain to the TSV. Note that the black dashed curve in (b) and (c) outlines the protrusion profile.

Fig. 2. (Color online) The process of $\mathrm{Cu}$ protrusion in a TSV under applied strain. The inserts on the right magnify the region highlighted by the white dashed box in the TSV structure on the left. The dislocation is denoted by the symbol $\perp$. The dimensionless time is (a) $t=8000$, (b) $t=9000$, (c) $t=10000$ and (d) $t=20000$.

Fig. 3. (Color online) The protrusion profiles of four TSVs, labeled TSV1 to TSV4, are denoted by black dashed lines. In the first three TSVs, two, three and four grains are placed in the middle layer, whereas one grain is placed in the bottom layer. In TSV4, four grains are placed in the middle layer, and two grains are placed in the bottom layer. The maximum protrusion heights of the four TSVs are plotted in the same figure.

Fig. 4. (Color online) (a) The protrusion profile is denoted by the black dashed curve. (b) The arrows show the trajectories of the atoms that lead to the protrusion in (a). A trajectory connects the initial location of an atom at $t=0$ to its corresponding location at $t=30000$ during the loading stage.

Fig. 5. (Color online) The relationship between $\ln \dot{\varepsilon}$ and $\ln \left(d^{-1}\right)$. Three types of loadings with different $\theta$ were applied to the TSV, i.e., $180^{\circ}$ (squares), $150^{\circ}$ (circles) and $120^{\circ}$ (triangles).

Fig. 6. (Color online) The relationship between the protrusion height and the TSV diameter. 
(a)

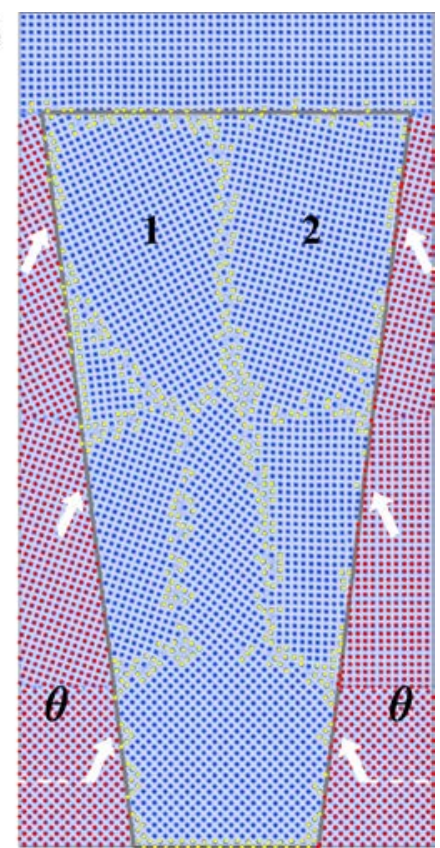

(b)

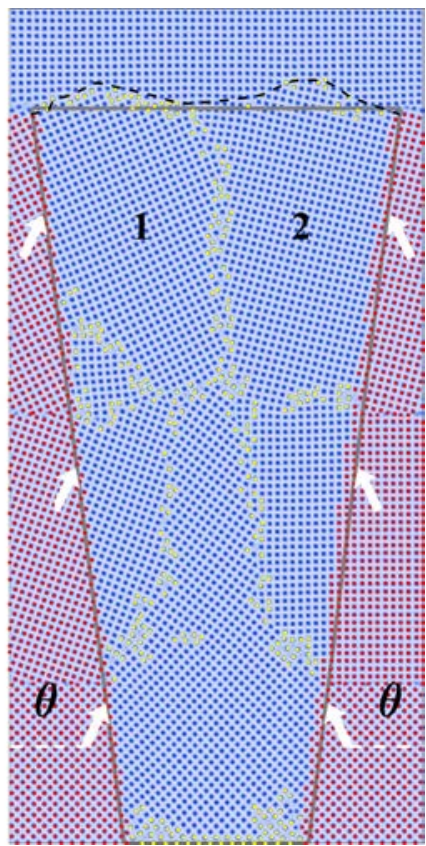

(c)

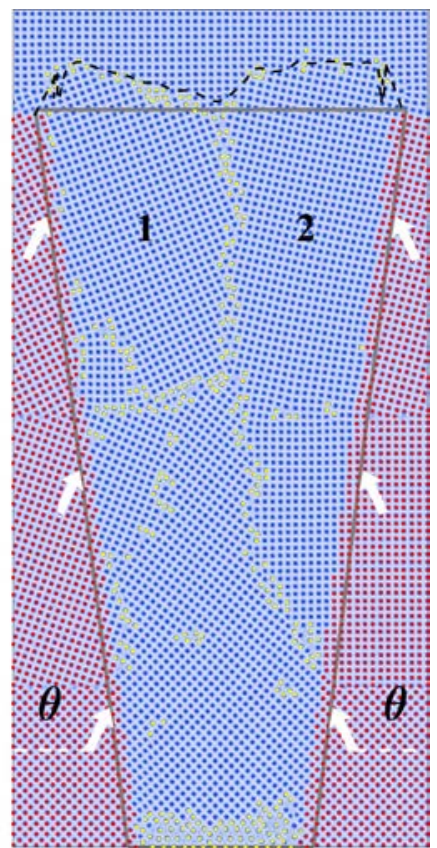

Fig. 1. (Color online) (a) The model configuration of a TSV structure. The edges of a trapezoid TSV at $t=0$ are outlined by solid gray lines. The parallel arrows indicate the direction of the strain applied to the TSV, which form an angle $\theta$ with the horizontal line. The defect atoms in the TSV are highlighted by the yellow dots. Note that the surface of TSV is not absolutely flat in (a) at the beginning of simulation, which is the result from a structure equilibration process between the TSV and the surrounding layers. To illustrate the applied loading, (b) and (c) show the snapshots of the TSV at $t=15000$ and $t=30000$ during the loading stage, respectively. The atoms in the external layers surrounding the left and right edges are highlighted by red dots. The red atoms are motivated to move by the "penalty term”, thus giving the effect of a compressive strain to the TSV. Note that the black dashed curve in (b) and (c) outlines the protrusion profile. 

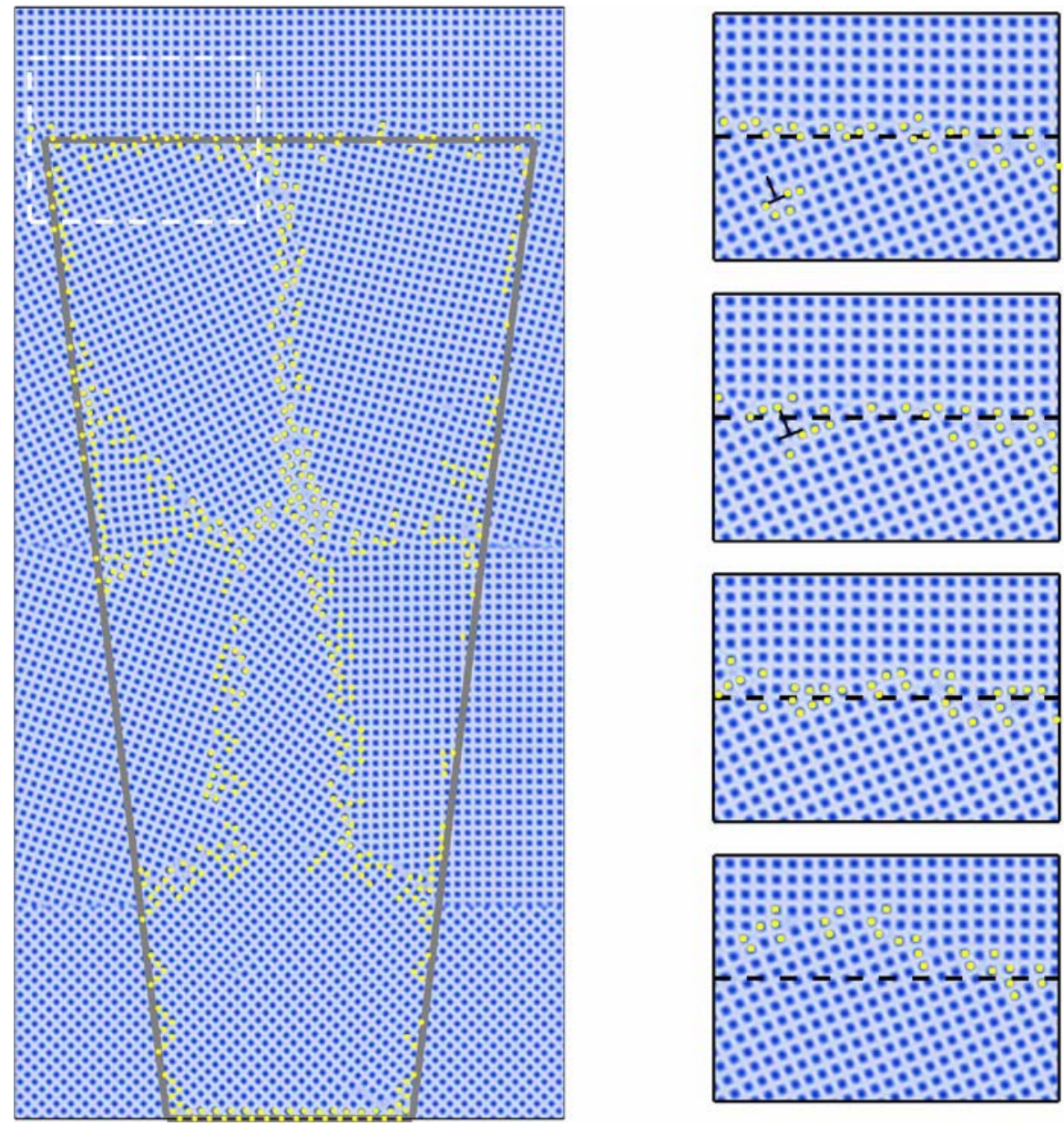

(a)

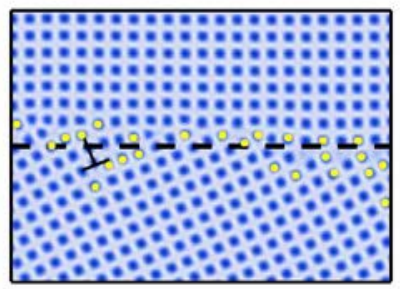

(b)

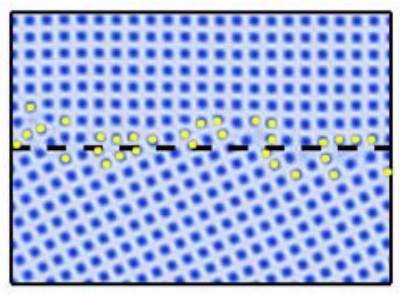

(c)

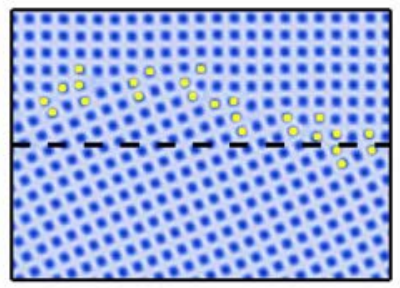

(d)

Fig. 2. (Color online) The process of Cu protrusion in a TSV under applied strain. The inserts on the right magnify the region highlighted by the white dashed box in the TSV structure on the left. The dislocation is denoted by the symbol $\perp$. The dimensionless time is (a) $t=8000$, (b) $t=9000$, (c) $t=10000$ and (d) $t=20000$. 


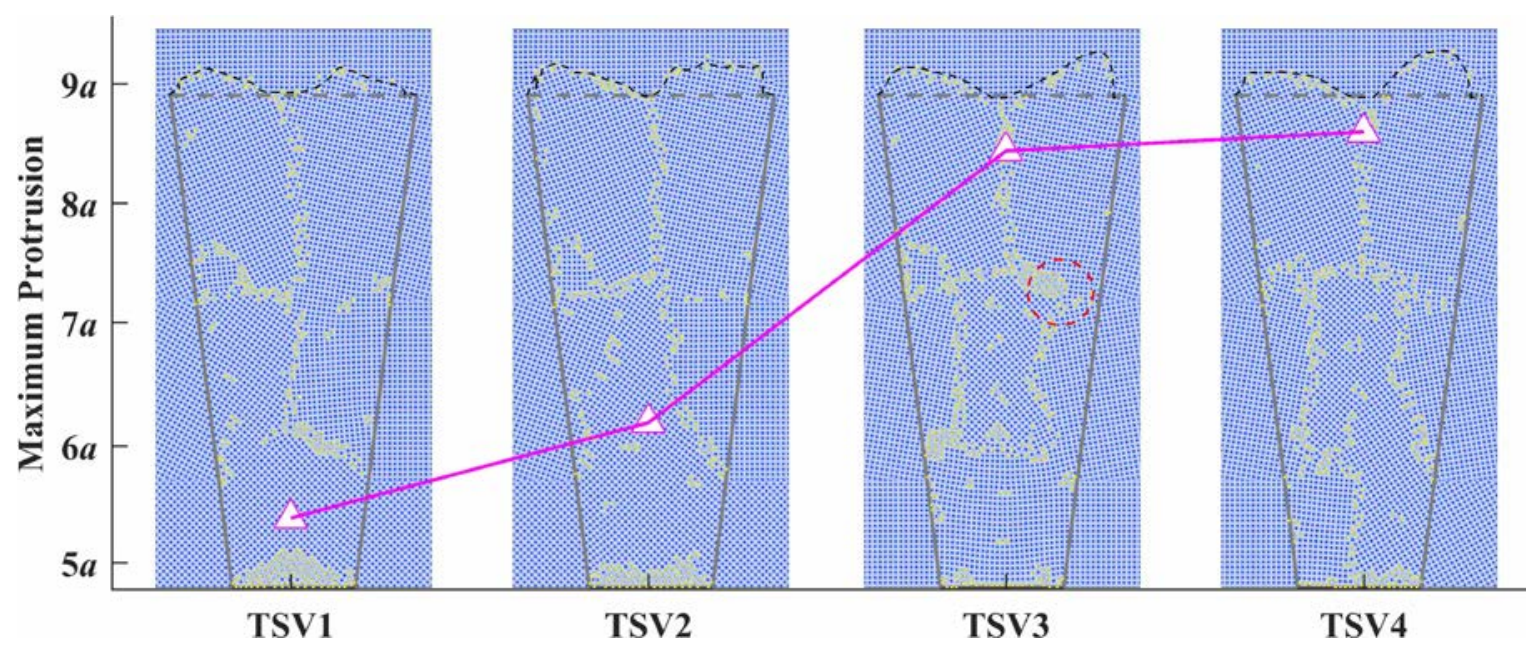

Fig. 3. (Color online) The protrusion profiles of four TSVs, labeled TSV1 to TSV4, are denoted by black dashed lines. In the first three TSVs, two, three and four grains are placed in the middle layer, whereas one grain is placed in the bottom layer. In TSV4, four grains are placed in the middle layer, and two grains are placed in the bottom layer. The maximum protrusion heights of the four TSVs are plotted in the same figure. 


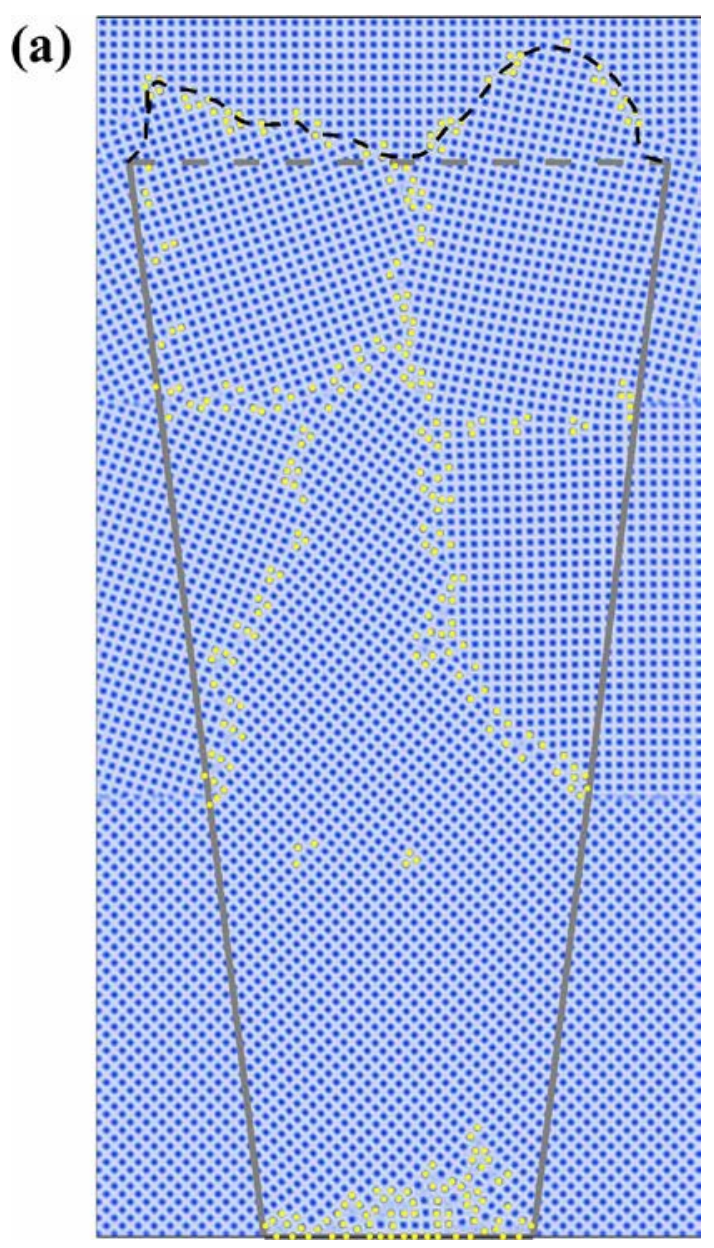

(b)

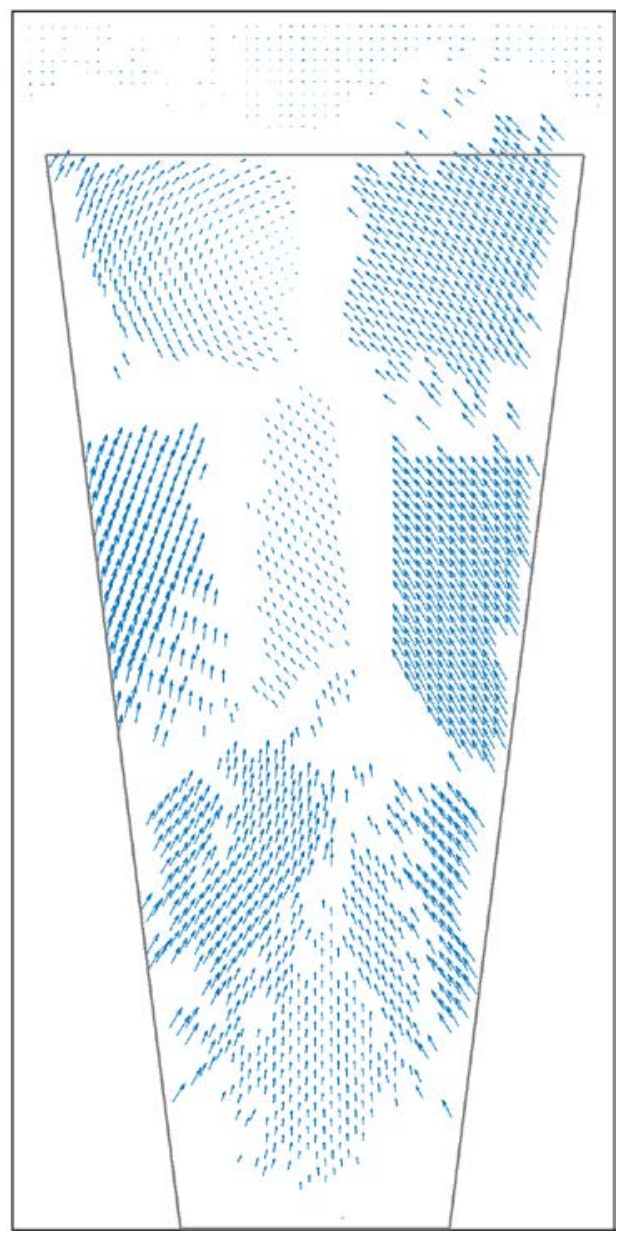

Fig. 4. (Color online) (a) The protrusion profile is denoted by the black dashed curve. (b) The arrows show the trajectories of the atoms that lead to the protrusion in (a). A trajectory connects the initial location of an atom at $t=0$ to its corresponding location at $t=30000$ during the loading stage. 


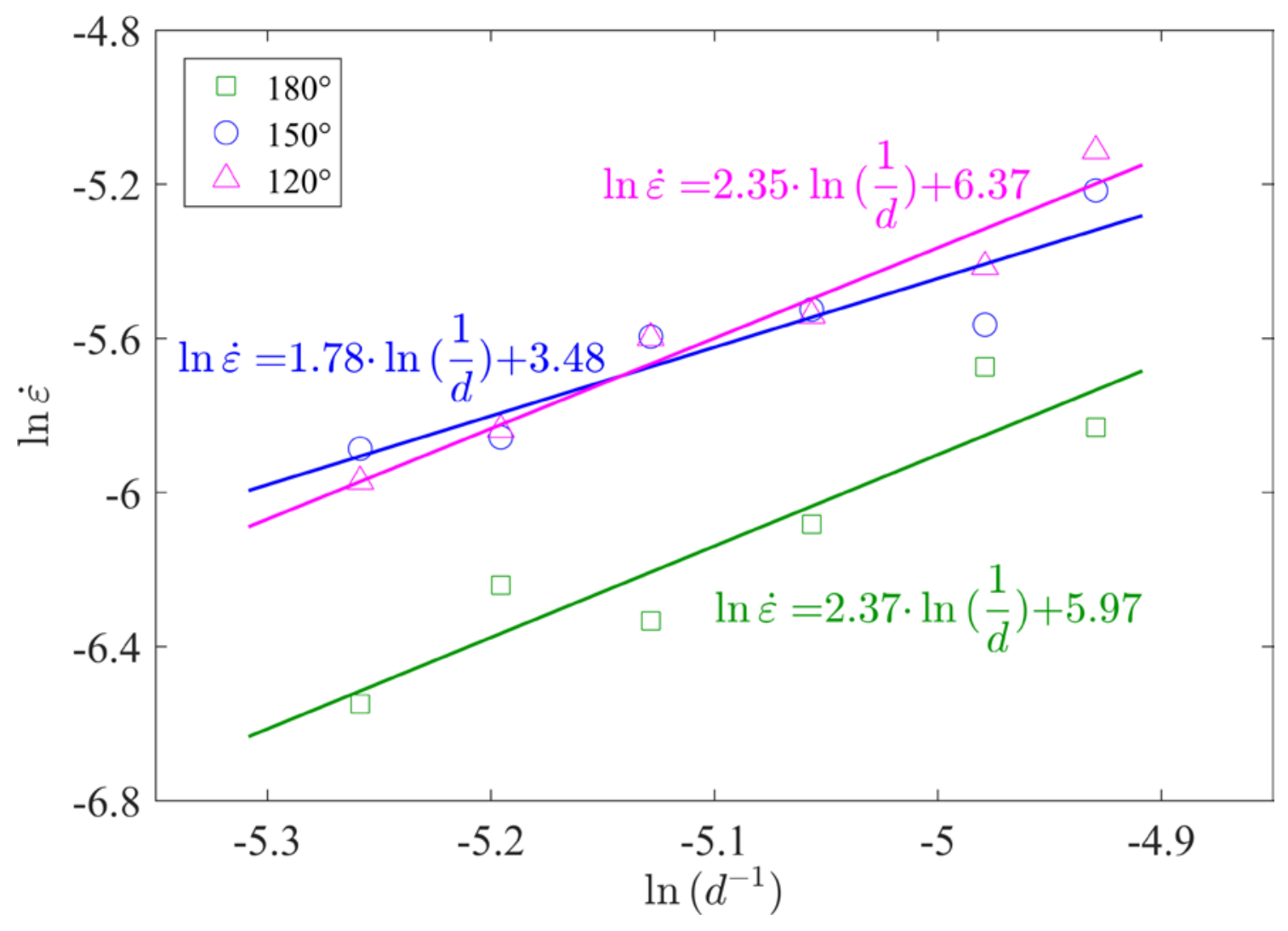

Fig. 5. (Color online) The relationship between $\ln \dot{\varepsilon}$ and $\ln \left(d^{-1}\right)$. Three types of loadings with different $\theta$ were applied to the TSV, i.e., $180^{\circ}$ (squares), $150^{\circ}$ (circles) and $120^{\circ}$ (triangles). 


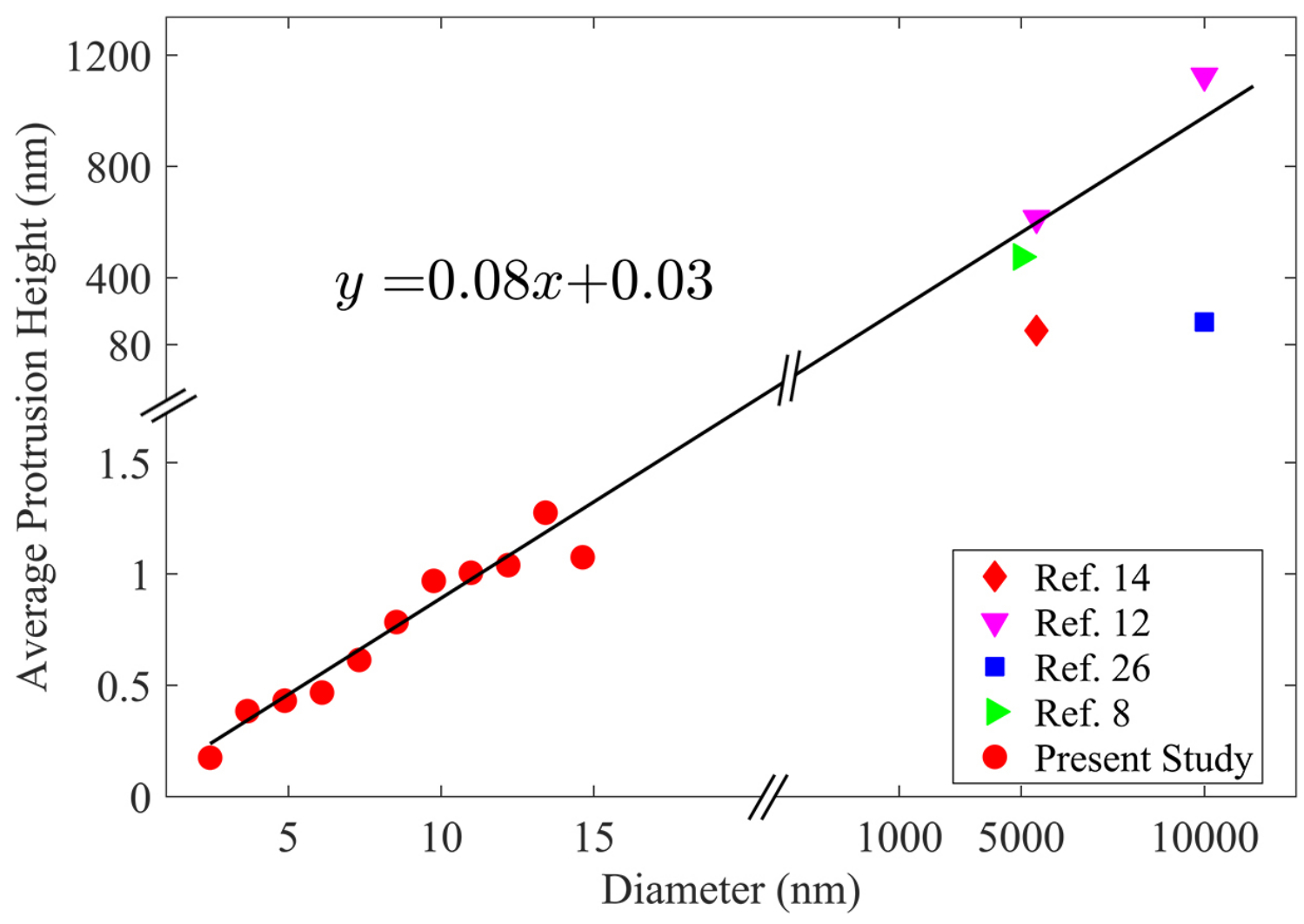

Fig. 6. (Color online) The relationship between the protrusion height and the TSV diameter. 